\title{
géotechnique et vibrations : effets sur l'environnement
}

\author{
Rapport général de \\ P. Chapot \\ Ingénieur, Laboratoire Régional des Ponts et Chaussées, Nancy \\ Ph. Weber \\ Professeur, École Nationale Supérieure des Techniques Industrielles et des Mines d'Alès
}

Dans la variété des sujets abordés, les diverses communications et interventions apportent des éléments de réponse aux trois questions centrales de ce thème :

- Par quelles relations la source d'une vibration et son environnement sont-ils liés?

- Comment l'environnement modifie-t-il la vibration?

- Comment déceler, puis quantifier, la dégradation que la vibration inflige à son environnement?

Douze communications ont donc èté présentées et discutées; de leur analyse, les rapporteurs généraux tirent tout d'abord deux commentaires préliminaires :

\section{Sur le choix d'un critère de nocivité}

Si aucune communication n'apporte d'éléments réellement nouveaux concernant la justification, théorique ou expérimentale, du critère de nocivité en vitesse particulaire, la plupart d'entre elles y font référence pour l'adopter: il semble en effet s'établir, ou se confirmer, un consensus sur le choix de ce critère, quitte à en moduler la valeur du seuil en fonction d'éléments liés à l'environement.

\section{Sur la prise en compte du paramètre temps}

Les sources de vibrations ont un caractère transitoire — généralement lié à la mise en ceuvre de l'explosif ou plus ou moins permanent - trafic routier. compactage, vibrofoncage. Or il apparait que le problème de l'influence de la durée de la víbration et son incidence sur la dégradation éventuelle de l'environnement, n'est guère abordé. Ainsi, les critères de nocivité auxquels la plupart des auteurs se réfèrent concernent les vibrations de tir; il s'agit de phénomènes qui sollicitent l'environnement durant quelques dixièmes de seconde à quelques secondes avec une récurrence qui peut varier de quelques tirs par jour à un tir isolé; s'il est très probable que la répétitivité des tirs doit avoir une influence sur l'environnement, aucun élément ne nous permet actuellement de I'appréhender; à la limite, vaut-il mieux fractionner un tir, quitte à solliciter plus longtemps l'environnement, ou au contraire lui infliger un seul train d'ondes d'amplitude plus élevée? Le choix d'un critère en vitesse peut conduire à retenir le premier terme de l'alternative, mais ce choix n'apparait pas nécessairement le meilleur.

En ce qui concerne les vibrations à caractère plus ou moins continu, il n'apparaît aucune donnée nouvelle concernant l'effet de "fatigue dynamique" sur les terrains et les structures sollicitées.

L'essentiel des développements auxquels ce thème a donné lieu est regroupé sous quatre chapitres :

- les critères de nocivité,

- la propagation des vibrations.

- la dispersion des mesures.

- la métrologie.

\section{Les critères de nocivité}

Si aucune des communications présentées ne traite de la détermination, ou de la justification d'un critère de nocivité, toutes celles qui concernent des travaux en zone construite font référence à celui de la vitesse particulaire maximale $[(12),(22),(31),(42)]$. Les nombreux exemples de travaux à l'explosif en site contraignant $[(31),(41),(42)]$, témoignent que la rêférence à ce critère s'est faite à la satisfaction des utilisateurs.

La référence à ce critère pose évidemment le problème du choix du seuil : malgré l'optimisme affiché dans les diverses communications, le choix du seuil apparait lié à une appréciation qualitative et intuitive des risques encourus, voire au contexte politique local ou à une estimation de la valeur des biens concernés. On notera à ce propos que la fourchette des valeurs seuil peut s'ouvrir de $5 \mathrm{~mm} / \mathrm{seconde}$ à $50 \mathrm{~mm} /$ seconde (12). 
Cette latitude dans le choix d'une valeur seuil a sans doute conduit à une trop grande prudence et à une limitation des charges unitaires à des valeurs beaucoup trop faibles, mais aucun élément ne permet de l'affirmer.

L'adoption quasi-générale de la vitesse maximum de vibration comme critère de nocivité est le résultat d'expériences systématiques effectuées en Suède, au Canada, aux Etats-Unis et en Tchécoslovaquie depuis une vingtaine d'années. Les expériences ont porté sur l'observation de dégâts provoqués sur des immeubles d'habitation courants et de hauteur limitée, par les vibrations dues à des tirs d'explosif, au total quelques dizaines de tirs sur quelques dizaines d'immeubles; les résultats de ces mesures ont permis de proposer des valeurs considérées comme "acceptables" pour les constructions : $70 \mathrm{~mm} / \mathrm{seconde}$ en Suède, $50 \mathrm{~mm} / \mathrm{se}$ conde en Amérique du Nord, 10 à 30 mm/seconde en Tchécoslovaquie.

Une étude plus fine, menée par le Bureau des Mines Américain a porté sur la discrimination entre un critère en accélération et un critère en vitesse particulaire : la corrélation (dégâts - critère en vitesse) apparaît effectivement moins dispersée que la corrélation (dégâts - critère en accélération).

Par la suite, le développement des mesures de vibrations sur les chantiers en zone plus ou moins urbanisée a amené à moduler les valeurs limites et, au fur et à mesure de la parution des publications sur le sujet, on voit diminuer les seuils de nocivité retenus par les divers auteurs. Un consensus s'établit néanmoins actuellement sur une fourchette comprise entre 10 et $30 \mathrm{~mm} /$ seconde dans la plupart des cas.

Certains pays ont transformé ces recommandations en normes mais il semble qu'elles reflètent davantage l'expérience ou la notorièté de quelques spécialistes: un projet de norme internationale s'est d'ailleurs soldé par un échec. Parallèlement à cette recherche sur la détermination d'un seuil, une réflexion s'est développée, visant à prendre en compte d'autres paramètres : caractéristiques des terrains de fondation, fréquence de la vibration (rappelons à ce propos que le choix d'un critère en vitesse particulaire constitue le corollaire du fait que les dégâts sont - ou semblent être indépendants des fréquences mises en jeu). Cette réflexion n'a jusqu'à présent pas abouti.

Les principales interrogations qui subsistent actuellement, et auxquelles les communications n'apportent que peu d'éléments concernent :

- l'adaptation des critères de nocivité à d'autres structures que les habitations de type standard. La protection des tunnels se fait actuellement par la même démarche que celle qui est utilisée pour les habitations, bien qu'il s'agisse de structures totalement différentes, et probablement susceptibles d'absorber sans dommage des vibrations d'un niveau nettement plus important:

- l'adaptation des critères à des sources autres qu'explosives : vibrofonçage, compactage;

- la prise en compte plus fine des paramètres géotechniques et des données de l'environnement: qualité des terrains de fondation;

- la prise en compte du paramètre temps selon le caractère permanent, temporaire, occasionnel ou isolé dans le temps, de la vibration.

Malgré ces lacunes qui doivent susciter autant de pistes de recherche, il convient de souligner le consensus qui se confirme sur le choix d'un critère de nocivité en vitesse particulaire et la restriction des valeurs seuils dans la fourchette $10-30 \mathrm{~mm} / \mathrm{seconde}$ pour les cas standards.

\section{La propagation des vibrations}

Deux approches différentes nous sont proposées par les intervenants. La première, purement empirique, consiste à modéliser l'amortissement de la vibration par une loi générale de la forme $V=K D^{-\alpha}$ et à déterminer expérimentalement les valeurs du coefficient d'amortissement $\alpha[(11$, Figure 1), (22, Figure 2), (42)] la seconde consiste à déterminer l'expression mathématique de la loi propagatoire $[(33),(34)]$ à partir d'hypothèses sur la source de vibration et le comportement du matériau; cette approche permet en particulier de mettre en lumière les contributions respectives des divers types d'ondes. Une étude sur modèle expérimental de laboratoire (13) montre que l'expression générale $V=K D^{-\alpha}$ est parfois mise en défaut.

Si la nature des terrains sollicités intervient à l'évidence dans l'expression de l'amortissement, aucune donnée nouvelle ne permet d'appréhender cette influence: la disposition des diaclases, la stratification et l'anisotrope du matériau, la géométrie locale des terrains, la présence éventuelle de terrains de fondation plus raides, ... doivent contribuer à l'expression générale de la loi d'amortissement; il semble que la dispersion des résultats expérimentaux masque ces contributions dans la plupart des cas. Les communications (21) et (22, Figure 3 ) font néanmoins référence à l'influence d'une tranchée comme facteur de réduction des vibrations.

Le coefficient d'amortissement $\alpha$ apparaît généralement compris entre 1,1 et $1,8[(11),(12)]$ pour des distances variant entre quelques mètres et quelques centaines de mètres. Cette ouverture importante de la fourchette traduit, outre une dispersion des résultats expérimentaux (nous reviendrons sur ce point ultérieurement), une évolution complexe des vibrations superficielles dans laquelle doit s'opérer graduellement, à mesure de leur progression dans les terrains, une modification dans la contribution des divers types d'ondes qui composent la vibration. On se heurte là à une question concernant la nature des vibrations: s'agit-il d'ondes de compression, de cisaillement, de surface? On sait que les deux premières s'estompent progressivement pour laisser la place, de façon prépondérante, aux ondes superficielles. La référence à un coefficient d'amortissement unique est sans doute illusoire.

L'approche théorique présentée dans les communications (33) et (34, Figure 4) n'est applicable qu'à une source excitative harmonique et ne peut rendre compte que de phénomènes permanents (trafic routier).

L'une des difficultés fondamentales pour le calcul de l'effet vibratoire induit par l'explosif réside dans l'impossibilité actuelle à modéliser l'interaction entre l'explosif et le milieu environnant: les phénomènes développés durant la détonation conduisent à une dégradation des terrains telle qu'elle résiste à toute approche scientifique.

Néanmoins, et sur un plan pratique, on peut noter avec satisfaction que les nombreuses mesures effectuées sur des sites variés conduisent à des expressions cohérentes de la relation entre vitesse maximale, 


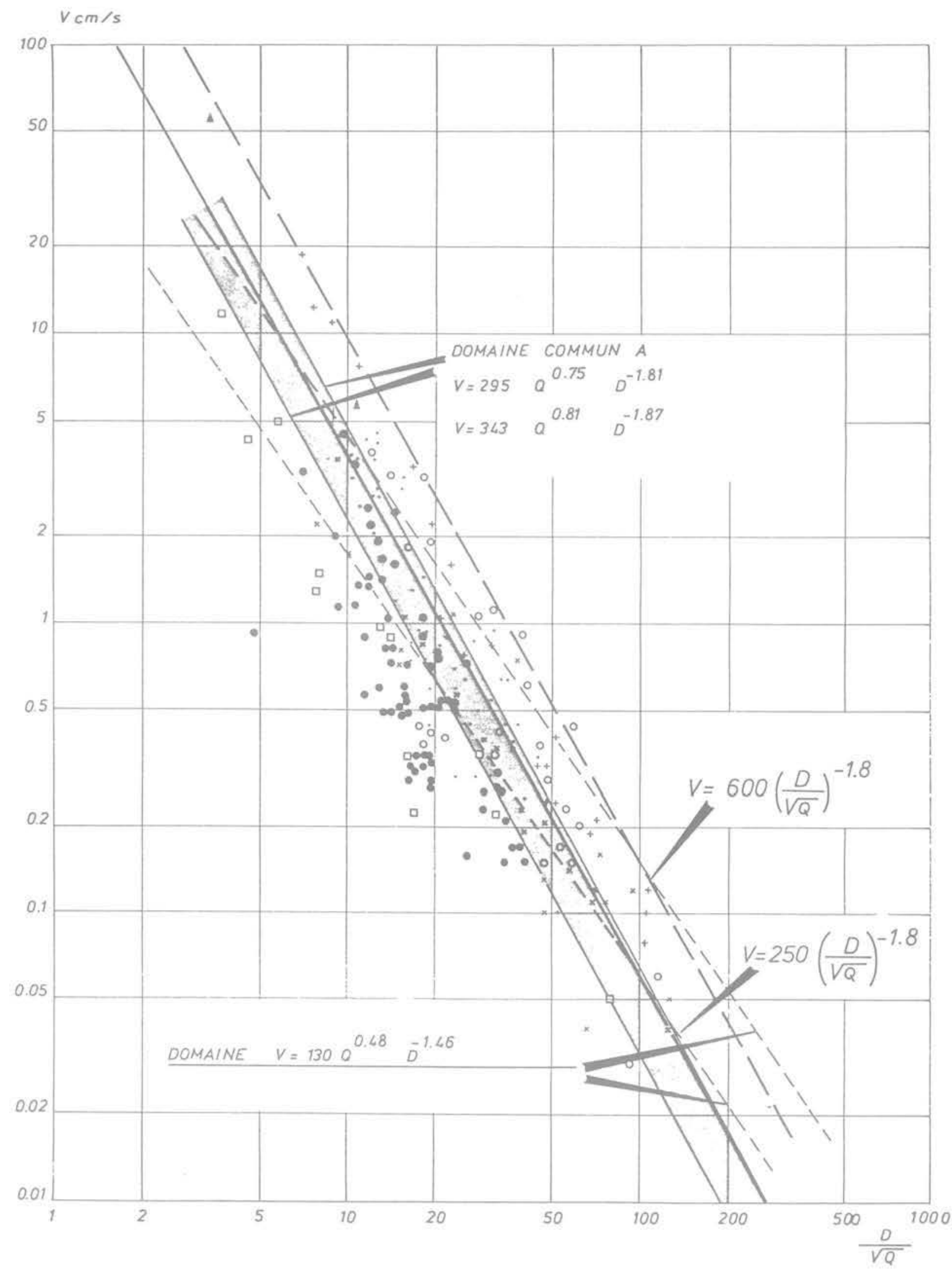

Fig. 1 Vitesse maximum de vibration en fonction de la distance réduite par la racine carrée de la charge

distance et charge unitaire [(11), (31), (42)]. Cette relation permet de définir, grossièrement, le niveau vibratoire escomptè sur un site donné, pour des tirs en surface.

La notion de * distance réduite " $(\mathrm{D} / \sqrt{\mathrm{Q}})$ est utilisée et permet de présenter la relation vitesse-distancecharge sous une forme simple (11, Figure 1).

\section{La dispersion des mesures}

Tous les intervenants soulignent l'importance de la dispersion des mesures de vibration consécutives au tir. Les raisons de cette dispersion sont multiples :

- Un essai de tir est par essence non reproductible, le site étant détruit chaque fois. 


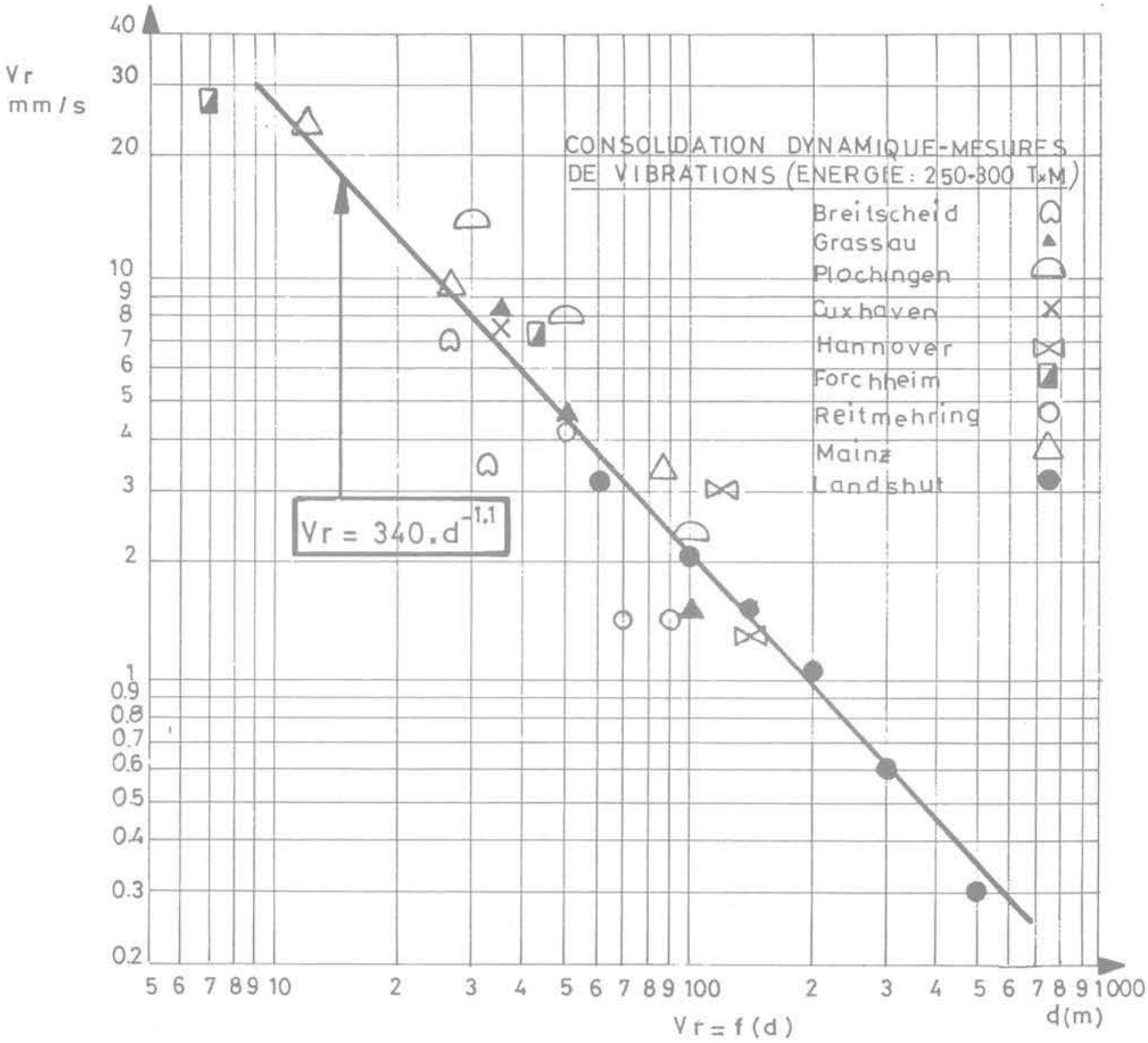

Fig. 2 Vitesse particulaire Vr en fonction de la distance $\alpha$ d'un impact (pour un choc de 200 à $300 t \times \mathrm{m}$ )

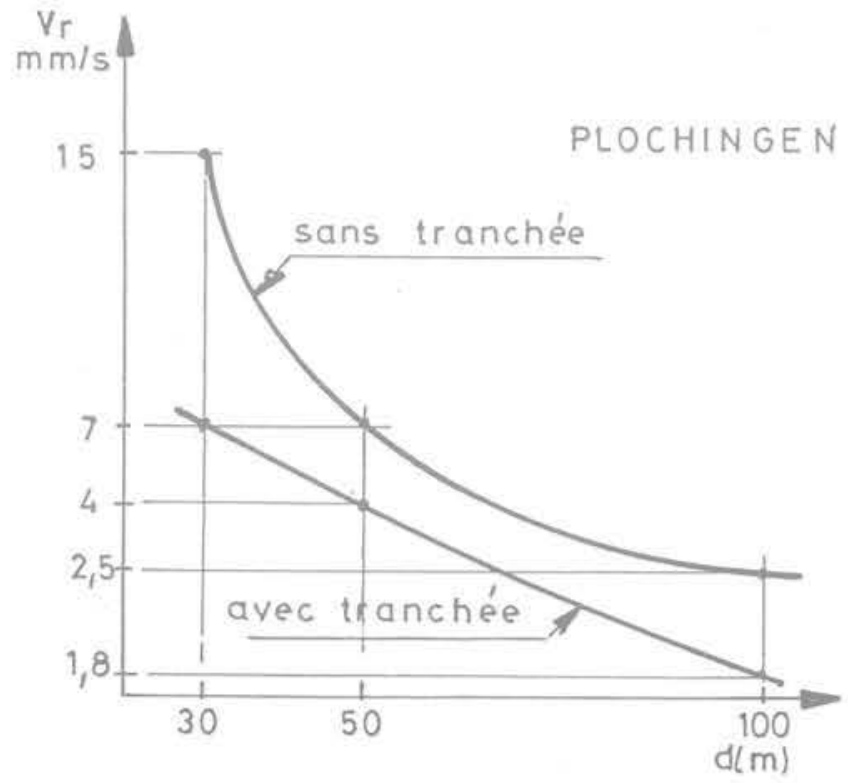

Fig. 3 Influence d'une tranchée de $2 \mathrm{~m}$ de profondeur environ sur les effets des ondes de Rayleigh
- Même dans des conditions de site parfaitement homogènes, et en réalisant le même plan de tir, on a noté un rapport 2,5 entre les amplitudes des vibrations extrémales. II semble en effet que le phénomène de détonation en site confiné ne soit pas reproductible.

- Enfin, dans le cas de tirs d'abattage, on peut avoir toutes les nuances entre un tir confiné parfaitement bourré et un tir à l'air libre (pétardage d'un bloc par exemple): dans le premier cas, l'énergie sismique générée dans le massif rocheux est maximale — de l'ordre de $20 \%$ de l'énergie totale libérée par la détonation -; dans le second cas, l'énergie sismique est quasi nulle. II semble d'ailleurs, au vu des résultats expérimentaux, que la dispersion des mesures de vibrations soit plus forte «vers le bas" que "vers le haut ": on conçoit en effet que, pour une quantité donnée d'explosif, il existe un maximum théorique d'énergie sismique transmise au terrain, et par conséquent une amplitude maximale de la vibration; par contre, le minimum théorique est nul.

- Soulignons enfin une dernière source de dispersion liée à l'instrumentation utilisée et à l'adaptation de la bande passante de la chaîne aux fréquences engendrées par le tir. 

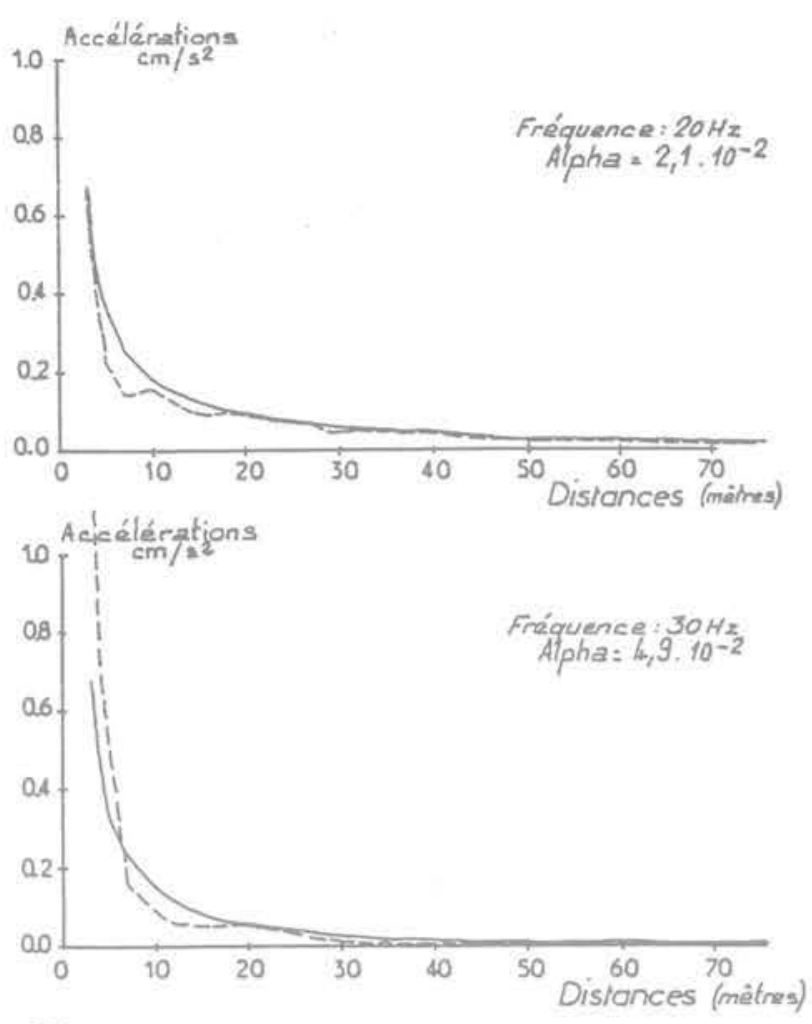
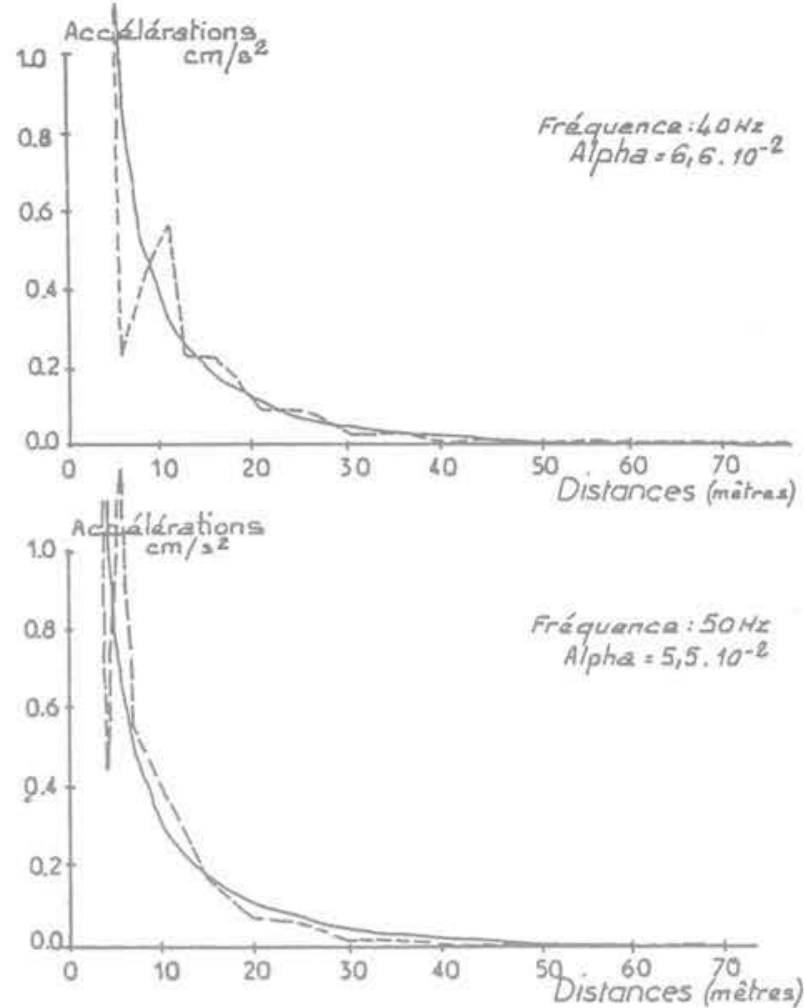

Fig. 4 Atténuation des ondes en fonction de la distance pour quelques fréquences sur du sable argileux - lissage du type $\left(W_{0} / d 1 / 2+W_{1} / d^{2}\right) \exp (-\alpha d)$

Cette dispersion des résultats expérimentaux inhérente à ce type de problèmes, masque partiellement l'origine des phénomènes observés et rend malaisée toute interprétation «fine" des résultats.

\section{La métrologie}

Sans revenir sur la grande variété des chaînes instrumentales utilisées, rappelons qu'elles sont de trois types :

- géophones + détecteur de crète;

- géophones + enregistreur et dépouillement en vitesse particulaire maximale et fréquence associée;

- géophones (ou accéléromètres) + enregistreur avec analyse complète du signal.

\section{Le choix de la bande passante}

Les premières mesures de vibration ont été faites, il y a une vingtaine d'années, sans précaution particulière concernant le choix de la bande passante; celle-ci étant de l'ordre de 10 hertz à quelques centaines de hertz.

II est apparu, à l'occasion d'enregistrements de tirs particuliers (tirs en terrains déconsolidés, enregistrement à grande distance) qu'il subsistait une énergie vibratoire significative à des fréquences de l'ordre de 1 hertz; inversement, lors d'enregistrements à faible distance du tir, il apparaît des fréquences supérieures à 1000 hertz. Dans quelle mesure les conclusions quant au choix d'un critère de nocivité, élaborées à partir d'enregistrements - probablement tronqués - sontelles valables? Comment intégrer ces nouvelles données instrumentales au niveau du choix du critère? Faute de données sur la contribution éventuelle de ces composantes à fréquence très élevée, ou très basse, à la nocivité de la vibration, il convient d'être très prudent : la tendance serait d'abaisser le seuil pour les composantes à basse fréquence, et de le rehausser pour les composantes à fréquence très élevée.

Une étude comparative des vibrations transcrites au même point par un accéléromètre de large bande passante et un géophone de fréquence de résonance 4,5 hertz (21) confirme la perte d'information par le géophone au-dessous de sa fréquence de résonance.

Si l'on désire une information complète sur la vibration, il y a donc lieu d'adapter le capteur au contenu spectral de la vibration et de noter que la réponse transitoire d'un géophone distord le signal d'entrée jusqu'à des fréquences de l'onde de 10 fois sa fréquence de résonance.

Une recherche approfondie doit être conduite, tant sur les performances des divers types de capteurs en régime transitoire, que sur le contenu spectral des vibrations.

\section{Le traitement de signal}

Deux types de traitement nous sont proposés :

- le traitement le plus rustique consiste à ne mesurer que I'amplitude de la vibration, et, éventuellement, la fréquence associée $[(11),(13),(31),(42)]$; ce traitement conduit directement à la définition du critère de nocivité en vitesse particulaire:

- un traitement plus complet est proposé dans les communications (21) et (32) notamment; il consiste à mettre en œuvre les techniques d'analyse harmonique et d'associer à la vibration son contenu spectral. Cette approche permet notamment :

- de corréler entre eux des signaux (21),

- de suivre l'évolution spectrale de la vibration (21),

- de définir une "signature sismique" d'un site déterminé (32), 
- de discriminer une vibration transitoire d'une pollution sismique ambiante (32),

- de définir une fonction de transfert d'une structure (21).

Cette approche doit ouvrir la voie à des progrès décisifs, en matière de connaissance des phénomènes vibratoires. Mais pour être réellement opérationnelle, elle devra être complétée par des données nouvelles concernant les mécanismes de dégradation des structures.

L'ensemble des communications et des interventions de ce thème "Géotechnique, vibrations et environnement " constitue une photographie actualisée de l'état des connaissances en la matière. Les rapporteurs souhaitent, en guise de conclusion, dégager les pistes de recherches qui leur paraissent les plus judicieuses et les plus efficaces, pour aboutir à la définition d'un critère d'endommagement dynamique des structures :
- Il conviendrait tout d'abord de mener une recherche sur la "fatigue dynamique" des structures et sur l'effet répétitif et cumulatif des sollicitations vibratoires.

- Puis d'amplifier l'effort actuellement mené afin de "décoder" et d'interpréter une vibration pour en dégager les paramètres réellement significatifs vis-àvis d'un éventuel endommagement.

- De développer toutes recherches, théoriques et expérimentales, sur les mécanismes de transfert d'énergie depuis la source excitatrice vers le milieu environnant.

- De poursuivre l'approche d'une définition probabilité du critère de nocivité, tenant compte du caractère dispersé et non répétitif de la vibration.

- Enfin et dans la mesure du possible, de mettre à disposition des divers laboratoires les informations et données de cas concrets afin de leur donner les moyens d'une interprétation de phénomènes d'endommagement réellement observés.

Références bibliographiques

Les références bibliographiques se rapportent aux communications présentées dans le thème II aux 2èmes journées nationales géotechniques, rassemblées dans le numéro 14 bis de la RFG. Le chiffre des dizaines des références renvoie au numéro du sous-thème et le chiffre des unités est le numéro d'crdre des articles dans le sous-thème. Le numéro 42 correspond à une présentation orale de MM. Chapot et Schwenzseier. 